\title{
CCD astrometry and instrumental $\Delta V$ photometry of visual double $\operatorname{stars}^{\star, \star \star}$
}

\author{
V. Differential measurements of pairs with angular separations $1^{\prime \prime}$ to $4^{\prime \prime}$ \\ D. Sinachopoulos ${ }^{1,2}$, A. Dapergolas ${ }^{2}$, E. van Dessel ${ }^{1}$, and E. Kontizas ${ }^{2}$ \\ 1 Royal Observatory of Belgium, Ringlaan 3, B-1180 Brussel, Belgium \\ 2 National Observatory of Athens, Lophos Nymphon, GR-11810, Greece
}

Received September 28, 1998; accepted February 16, 1999

\begin{abstract}
We present angular separations, position angles and $\Delta V$ instrumental magnitude differences of 83 visual double stars with angular separations around two arcseconds. Our observations were made by means of a CCD detector attached to the Kryonerion $1.2 \mathrm{~m}$ telescope. 12 of them are common proper motion stars and their components should therefore be stars of common origin.
\end{abstract}

Key words: stars: binaries: visual — astrometry

\section{Introduction}

After the publication of the HIPPARCOS catalogue, containing the results of this astrometric mission (ESA, 1997), one can but notice how scanty our data on visual binaries are, both from the photometric as from the astrometric point of view. Especially data on couples with separations in the order of a few arcseconds do not allow to evaluate their significance, or to make reliable statistics. It is therefore evident to us that ground-based observations of visual double stars with angular separations of a few arcseconds have to be continued; CCD's (not in use for very long yet, compared to the history of visual binary observing) provide new data of high reliability; statistical treatment of the new observations combined with the available old ones provides a strong tool for studying relative proper motions and thus to decide upon the nature of the objects (cf. Brosche \& Sinachopoulos 1988 and Brosche et al. 1991).

Send offprint requests to: D. Sinachopoulos

* Based on observations made at Kryonerion Observatory, Greece.

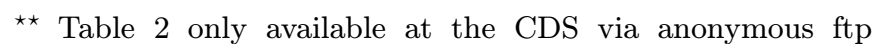
to cdsarc.u-strasbg.fr (130.79.128.5) or via http://cdsweb.ustrasbg.fr/Abstract.html
The present results are a continuation of the observing programme we initiated more than ten years ago (Sinachopoulos 1988, Paper I). In the discussion we shall have particular interest as to what fraction of these relatively wide-separation stars show significant motion after (at least) several decades. Also compared to the previous articles in this series of papers, the objects chosen are closer to the possibilities the reduction technique affords, linked to the limiting seeing conditions; some additional comments concerning the reduction procedure are given in Sect. 3.1. Experience through all these years has shown that CCD detectors are very much suited for these observations, as e.g. Sinachopoulos et al. (1988) claimed.

\section{The observations}

\subsection{Site and instrumentation}

Our observations were carried out at the Kryonerion Observatory of the National Observatory of Athens, Greece, in four clear but not really photometric nights, between June 25 and 28, 1998.

We used the Photometrics CCD camera of the observatory, attached to the Cassegrain focus of its 1.2 meter telescope. Its back illuminated and thinned chip has $516 \times 516$ square pixels of $24 \mu$ size. We refer to D. Sinachopoulos et al. (1998) for a detailed discussion of the CCD chip. One exposure per CCD frame was made, each having a duration of a few seconds. Four sequential CCD frames were taken per double star observed.

A Bessel $V$ filter was used for all exposures. We observed targets with both components fainter than magnitude 8 . Given the relatively small angular size of the pixel and seeing conditions, as they are discussed below, we did not need to use a density filter.

Most observations were made very close to meridian, in order to minimize the effect of atmospheric refraction. 


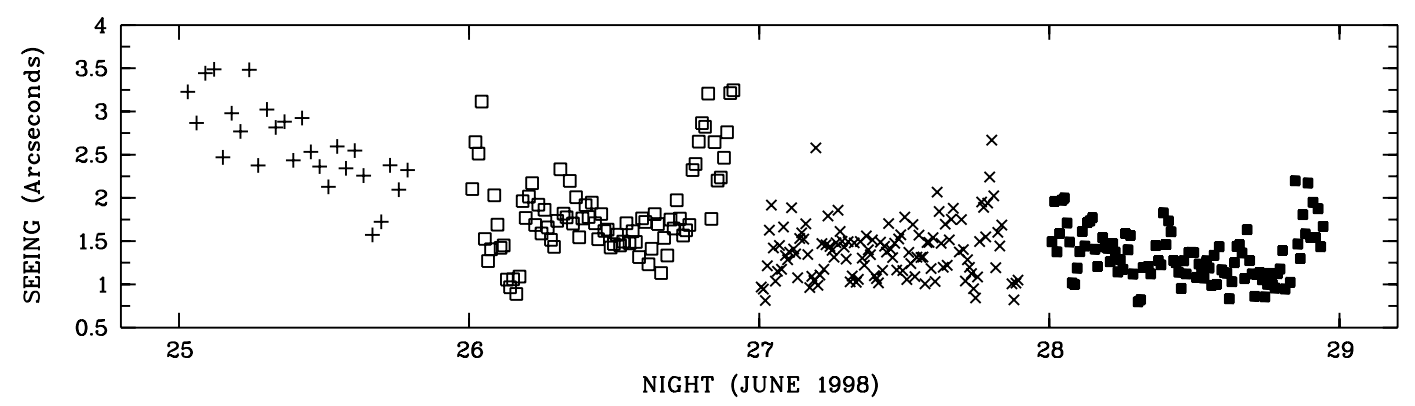

Fig. 1. Seeing (FWHM) in four nights

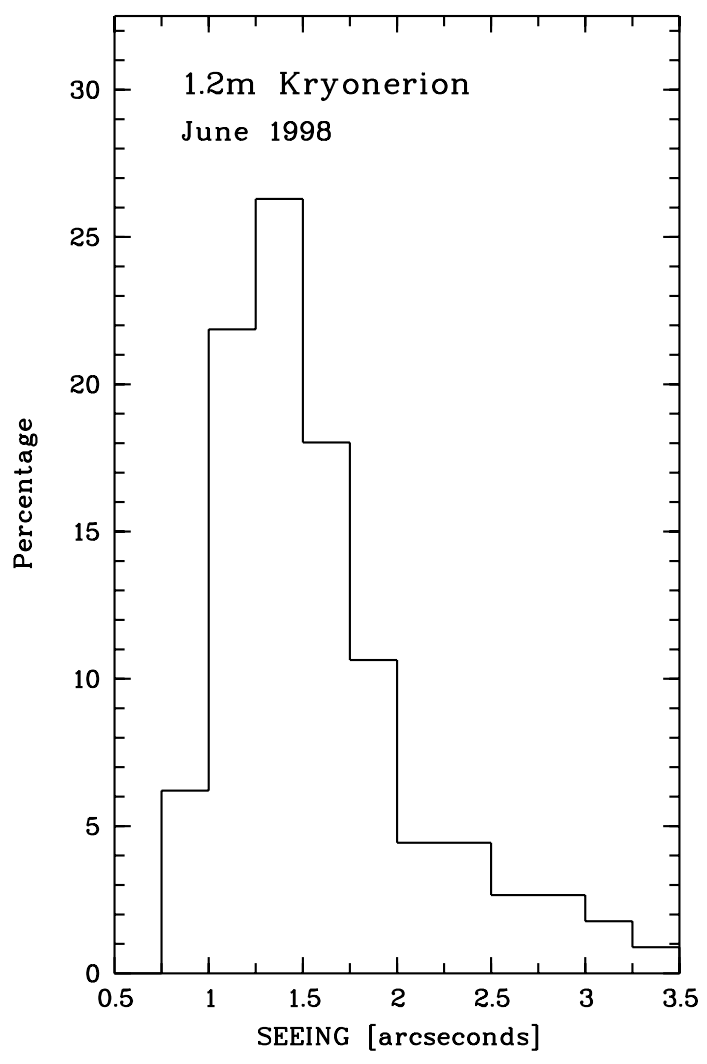

Fig. 2. Seeing (FWHM) measurements

The reduced data of our observations in the two nights of June 27 and 28 indicate that these two nights were partially photometric.

\subsection{Seeing conditions}

We monitored the dome seeing during our observations, defined as the full width at half maximum (FWHM) of a well exposed, and therefore symmetric, stellar image on a CCD frame. It was found $2.5 \pm 0.5$ arcseconds in the first night, $1.8 \pm 0.5$ in the second, $1.4 \pm 0.3$ in the third, and $1.3 \pm 0.3$ in the last one. Figure 1 presents seeing development through the four consecutive nights and Fig. 2 its distribution in the same period. Remark that the pixel size is 0.30 arcseconds.

Temperature changed only up to two degrees around the same mean value (19 degrees) each night.

\section{Data reduction}

More than 350 CCD frames were taken during the four nights. The data reduction was performed by using the ESO-MIDAS image processing software. It evidently included a BIAS offset subtraction and a flat-field correction has also been performed for "cleaning" the observations.

\subsection{The used method of PSF fitting the data}

Usually, only the two components of the visual binary observed were on the CCD frames. Obviously, the images of two components overlapped strongly. Therefore, we fitted simultaneously two MOFFAT profiles to both components in order to obtain the instrumental component magnitude difference and relative positions. This method is described by Cuypers (1997) in detail. Our FORTRAN programme used here for the data reduction makes use of this method. In addition, it detects the two components on the CCD frame automatically and calculates accurate initial values of the double MOFFAT function, needed for the leastsquare fitting, automatically as well.

Since the angular separation of the components is of a few arcseconds only, their images have identical shapes. So, the two fitted MOFFAT functions should have the following profile determining parameters in common: $b$-that is related to the FWHM-, e and f: parameters of the ellipticity, and q: the exponent of the MOFFAT function (see Cuypers 1997). Figure 3 shows the strong correlation between seeing and the exponent of the MOFFAT function "q". The symbols used in Fig. 3 have the same meaning as the ones of Fig. 1, corresponding to the four different nights. The corresponding slope of the linear regression was $0.70 \pm 0.05$ for the second night, very significantly different from that of the third $(0.35 \pm 0.04)$ and the fourth $(0.48 \pm 0.04)$. 


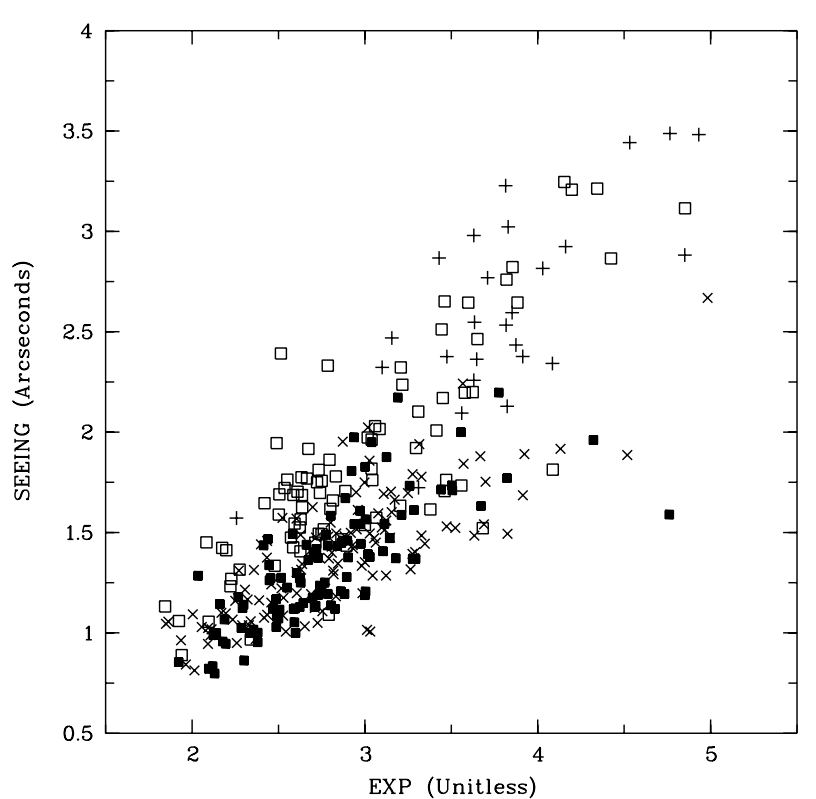

Fig. 3. Correlation between seeing and the exponent of the MOFFAT function "q"

Table 1. CCD scale determination using HIPPARCOS stars

\begin{tabular}{|c|c|c|c|c|c|}
\hline \multicolumn{2}{|c|}{ HIPPARCOS } & \multirow{3}{*}{$\frac{\begin{array}{c}\rho_{\mathrm{HIP}} \\
{\left[{ }^{\prime \prime}\right]}\end{array}}{16.24}$} & \multirow{2}{*}{\multicolumn{2}{|c|}{$\begin{array}{c}\rho_{\mathrm{CCD}} \\
\text { [pixels] }\end{array}$}} & \multirow{3}{*}{$\begin{array}{c}\text { CCD scale } \\
{[" / \text { pixel }]}\end{array}$} \\
\hline $\mathrm{B}$ & $\mathrm{A}$ & & & & \\
\hline 100687 & 100690 & & 53.81 & .01 & \\
\hline 111276 & 111273 & 14.49 & 48.48 & .02 & 0.2990 \\
\hline 77503 & 77504 & 13.86 & 45.95 & .02 & 0.3017 \\
\hline 83744 & 83747 & 18.34 & 60.73 & .03 & 0.3021 \\
\hline 93600 & 93601 & 11.42 & 37.92 & .02 & 0.3013 \\
\hline
\end{tabular}

\section{2. $C C D$ scale determination}

We used the five HIPPARCOS (ESA, 1997) star pairs listed in Table 1 below in order to extract the CCD scale. The first two columns contain the two HIPPARCOS stars identification of the wide visual double star components. HIPPARCOS celestial $(\alpha, \delta)$ coordinates of these stars have been transformed first to actual ones of the epoch of observations (1998.48). Then, the corresponding angular separation of the pair in arcseconds has been calculated (third column). Column four contains the corresponding mean values of angular separation in pixels as resulted from the chip instrumental astrometry together with its uncertainty. Since atmospheric conditions (temperature and air pressure) were quite stable during observations, these values are mean standard deviations of all the individual separations measured in different nights. From column two and three the corresponding scale of the CCD chip has been calculated (column five). From these five individual scale values we obtained the final unweighted mean value of the scale $m=0.30118 \pm 0.00056$ arcseconds/pixel.
One may remark that the uncertainies of angular separations due to the instrumental on CCD chip are around 0.02 pixels, equivalent to 0.006 arcseconds. Since our astrometric standard double stars have an angular separation of around 50 pixels, we expected an internal scale accuracy (due to the used CCD pixel size and the telescope focal length) around 0.00006 arcseconds/pixel. It is then clear that the resulted uncertainty of the scale $(0.00056$ arcseconds/pixel) is dominated by the HIPPARCOS catalogue uncertainties. This is mainly due to the rapid deterioration of the proper motion accuracies in this catalogue with time, which is the reason, in our case, for the relatively high uncertainty of the scale.

We calculated the same using data from the Brosche \& Sinachopoulos $(1988,1989)$ catalogues and we found a scale $m=0.3046 \pm 0.0025$ arcseconds/pixel. This value is four times less accurate than the value of CCD scale based on the HIPPARCOS results and thus not used.

\subsection{Position angle determination}

Trails of up to ten stars near the celestial equator per night for defining the direction of the right ascension on the CCD chip were taken. We determined from them that this zero point is $\theta=-0.34 \pm 0.02$ degrees. We added this value to the instrumental position angle.

The precision achieved in both the angular separation and the position angle has been often discussed in our previous papers of this series, especially by Sinachopoulos \& Seggewiss (1990, Paper II), and by Nakos et al. (1995, Paper IV, and 1997).

It is nevertheless necessary to mention that we took $\sigma_{\mathrm{r}}(z)=0.004$ as an approximation of the mean error due to refraction for the used $V$ filter. According to Herzsprung (1920), this value of $\sigma_{r}(z)$ is the expected difference in position $\delta(z)$ due to refraction between an Atype star and a K-type star at zenith distance $z=45^{\circ}$ for observations with yellow sensitive plates, which correspond to the $V$ filter we used (see also in Sinachopoulos \& Seggewiss 1990, for more details). Since we always observed at smaller zenith distances and the colour difference between the two components of our targets is probably not often that large, we consider the value of $\sigma_{\mathrm{r}}(z)$ we adopted to be always larger than the correct value.

\section{The results and discussion}

We present our results in Table 3. The first two columns contain the coordinates of the primaries for epoch 2000 . Next column contains the Durchmusterung designation of the primaries as listed in WDS (Worley \& Douglass 1984). The next two columns contain the observed instrumental magnitude difference between the two components of the binary and its accuracy, $\Delta V$ and $\sigma_{\Delta V}$. The following four 
Table 3. Common proper motion binaries in our sample

\begin{tabular}{lrrrrrr}
\hline \multicolumn{2}{c}{ BD } & $\rho_{\text {Det. }}$ & $\rho_{1998.5}$ & $\theta_{\text {Det. }}$ & $\theta_{1998.5}$ & Epoch $_{\text {Det. }}$ \\
\hline N13 & 3144 & 3.2 & 3.29 & 145 & 144.64 & 1897 \\
N50 & 2324 & 2.6 & 2.55 & 45 & 44.26 & 1901 \\
N19 & 3243 & 3.1 & 3.18 & 204 & 204.29 & 1909 \\
N45 & 2574 & 2.7 & 2.78 & 296 & 296.77 & 1893 \\
N20 & 3750 & 2.5 & 2.45 & 194 & 194.53 & 1890 \\
N36 & 3430 & 2.5 & 2.56 & 2 & 2.71 & 1901 \\
N21 & 3726 & 2.6 & 2.61 & 325 & 324.35 & 1831 \\
N19 & 4183 & 3.4 & 3.37 & 45 & 45.38 & 1901 \\
N34 & 3771 & 2.5 & 2.50 & 332 & 331.88 & 1970 \\
N32 & 3883 & 3.1 & 3.13 & 262 & 262.07 & 1831 \\
N30 & 4227 & 3.4 & 3.42 & 135 & 134.54 & 1906 \\
N52 & 2963 & 3.4 & 3.45 & 325 & 325.07 & 1876 \\
\hline
\end{tabular}

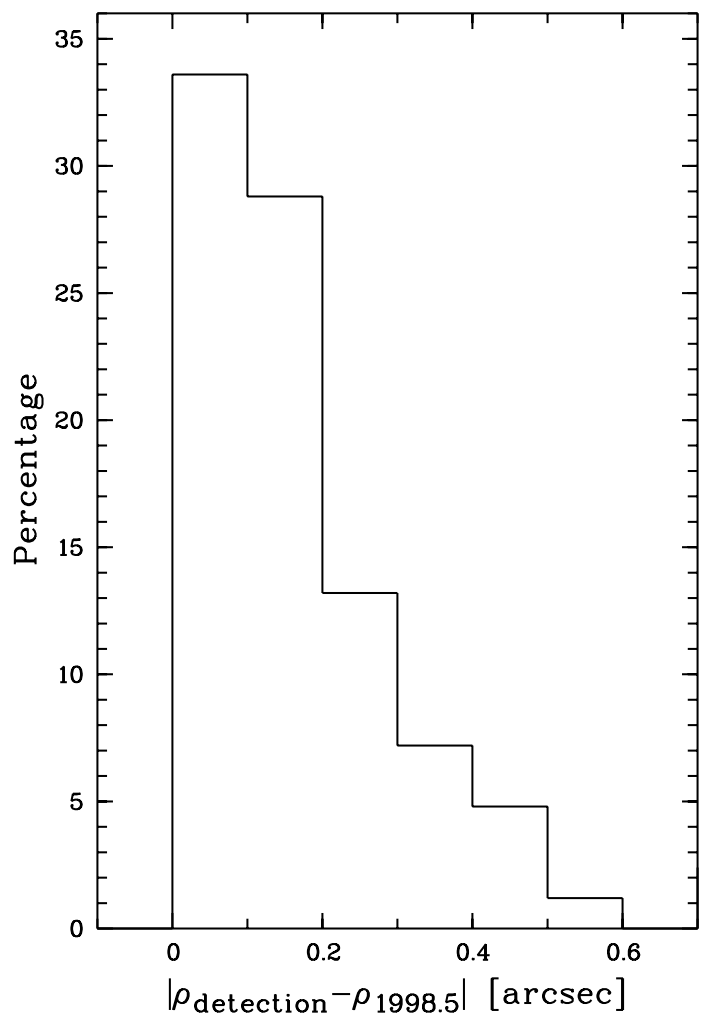

Fig. 4. Absolute values of differences in angular separations since the detection of the binary

columns contain angular separation $\rho^{\prime \prime}$, its accuracy $\sigma_{\rho^{\prime \prime}}$ in arcseconds, and the position angle $\theta$ with its accuracy $\sigma_{\theta}$ in degrees. All accuracies of the mean values always refer to four consecutive exposures of the same binary. The last column contains the epoch of the observation in years after 1900.0.

The histogram of Fig. 4 shows that $33 \%$ of the 83 observed binaries have an angular separation different by less than 0 "' 1 or $5 \%$ of the initially observed -at the epoch of the detection of the binary- angular separation several decades ago. Additional $29 \%$ show changes in the range of

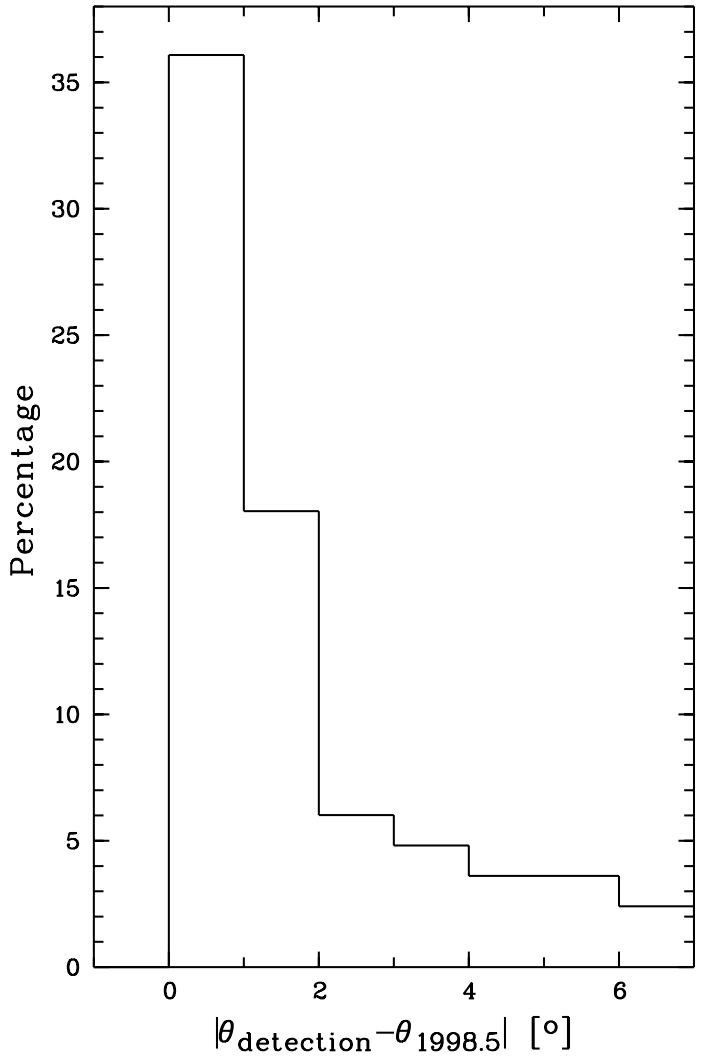

Fig. 5. Absolute values of differences in position angle since the detection of the binary

$5 \%$ to $10 \%$. ( $\left.0^{\prime \prime} \cdot 1-0,2\right)$. Most of them (73 or $88 \%$ ) show $\left|\rho_{\text {detection }}-\rho_{1998.5}\right| \leq 0$.".5. Taken into account that WDS lists $\rho_{\text {detection }}$ with a precision of 0.1 arcseconds and that the first double star observation is several decades old or even from last century -thus less accurate due to the technology used-, most of our targets show practically even now the same angular separation as at the epoch of their detection.

Similarly, Fig. 5 shows that $36 \%$ of them change their position angle less than $1.0^{\circ}$ (the precision of the position angle listed in WDS) in all these decades since their detection. Furthermore, 57 of them (or 70\%) show $\left|\theta_{\text {detection }}-\theta_{1998.5}\right| \leq 5^{\circ}$.

Table 2 shows 12 double stars in the sample which show almost the same relative positions as at the epoch of their detection. The first column contains the BD number of the primary, columns two and four the angular separation and the position angle of the components at the epoch of the detection as listed in WDS. This epoch is shown in column six. Finally, corresponding relative positions of the present work are listed in columns three and five respectively.

Since the epoch of double star detection listed in column six is usually many decades ago, these double stars must be common proper motion double stars and thus very strong candidates to be physical binaries. More 
astrophysical data should be gathered on them; we will be able to execute a photometric programme to this purpose thanks to the availability of the Kryonerion telescope.

Acknowledgements. This research was partially carried out in the framework of the project "Pôles d' Attraction Interuniversitaires" P4/05, initiated and financed by the Belgian Federal Scientific Services (DWTC/SSTC).

\section{References}

Brosche P., Sinachopoulos D., 1988, Astroph. Space Sci. 142 $255-258$

Brosche P., Sinachopoulos D., 1989, Bull. Inform. CDS 36

Brosche P., Denis-Karafistan A.I., Sinachopoulos D., 1991, A\&A 253, 113-118

Cuypers J., 1997, Proceedings of the Conference "Visual Double Stars": Formation, Dynamics and Evolutionary
Tracks, 35, J.A. Docobo, et al., Kluwer Academic Publ., The Netherland

ESA 1997, The HIPPARCOS catalogue, ESA SP-1200

Herzsprung E., 1920, Publ. Astrophys. Obs. Potsdam 24, No. 75

Nakos Th., Sinachopoulos D., van Dessel E., 1995, A\&AS 112, 453

Nakos Th., Sinachopoulos D., van Dessel E., 1997, A\&AS 124, 353

Sinachopoulos D., Nicklas H., Geffert M., 1988, Astrophys. Space Sci. 142, 227-230,

Sinachopoulos D., 1988, A\&AS 76, 189-193

Sinachopoulos D., Seggewiss W., 1990, A\&AS 83, 245

Sinachopoulos D., Dapergolas A., Kontizas E., 1998, Memoirs of the National Observatory of Athens, Ser. I, No. 36

Worley C.E., Douglass G.G., 1984, The Washington Visual Double Star Catalogue, Publ. U.S. Naval Obs. (magnetic tape) 